\title{
Evaluation of Anal Manometric Findings in Haemorrhoids before and after Haemorrhoidectomy
}

M.A.Khalil, E.M.Oraby, M.A.El Sayed and M.A.AbdelSamie

General Surgery Dept., Faculty of Medicine, Benha Univ., Benha, Egypt

E-Mail:Mostafa2356@gmail.com

\begin{abstract}
The anal pressure abnormalities in haemorrhiods before and after surgery were studied by an ano-rectal manometric technique. Fifty consecutive patients (32 men, 18 women; mean age 42.96 years) with symptomatic haemorrhoids (grades III and IV) and different degrees of severity were submitted to this study. Schedule excisional haemorrhoidectomy might have been performed. Those butt-centric resting weight were monitored previously, then at then afterward two months for surgery when those wounds required mended. There might have been critical diminishing in the mean resting weight "around instances post-operative over pre-operative. Sodergren haemorrhoid manifestation seriousness scoring framework were used to quantify those seriousness about haemorrhoidal symptoms, as stated by this scoring framework there were no huge Contrast between Different degrees for seriousness in regards should manometrical effects (resting butt-centric pressure).
\end{abstract}

Keywords: Anal, manometric, Pressure, Haemorrhoidectomy, Severity.

\section{Introduction}

Aged references on symptomatic haemorrhoidal illness go back many quite some time Furthermore could make discovered for right on time Egyptian, Babylonian, What's more Greek scripts. Those principal referred to specify about this condition is from an egyptian papyrus to $1700 \mathrm{bc}$ [1].

Those term about haemorrhoids as a rule identifies indications Furthermore side effects Emulating distal uprooting of butt-centric pads initiated by discontinuity Furthermore degenerative progressions in the submucosal tissue of the butt-centric canafistula. They influence a large number of individuals around the world, and representable An major medicinal What's more socio-investment issue [2].

An number of factors would accepted will assume a part Previously, haemorrhoids, including unpredictable inside habits, expanded intra-abdominal pressure, a nonattendance about valves inside the haemorrhoidal veins, and maturing [3].

Haemorrhoidal manifestations are accepted to effect The point when butt-centric pads slide downwards alternately The point when venous weight will be exorbitantly expanded. Expanded interior and outside butt-centric sphincter weight might additionally make included to haemorrhoid indications. The part of raised butt-centric canafistula weight in the pathophysiology about hemorrhoids need been the subject from claiming a great deal study and discussion [4,5].

Resting butt-centric weight will be generally because of those withdrawal of the interior sphincter. The inside butt-centric sphincter tone arm supplies 55\% will $85 \%$ of the resting weight. Those outer sphincter will be for a state of tonic stress fractional contraction, considerably Throughout rest What's more contributes over $15 \%$ of the resting weight [6].

Large portions investigators bring accounted for abnormal butt-centric sphincter manometric discoveries done patients for haemorrhoids, inasmuch as for best a couple reports might have been secondary sphincter weight not discovered [7].

A few investigations news person that helter skelter butt-centric sphincter weight will be an essential reason for haemorrhoidal disease, whichever butt-centric dilatation or parallel inside sphincterotomy need been advocated with treat its pathogenesis What's more lessen postoperative difficulties Furthermore agony [8].

\section{Subjects and methods \\ The study data}

This randomized prospective ponder might have been outlined on assess patients with haemorrhoids Toward ano-rectal manometry. A tolerant characteristic, symptoms, manometric discoveries might have been recorded in Benha college healing center following bringing the Regard of the moral council for general surgery Branch. Educated assent might have been taken from every one patients incorporated in the contemplate.

Fifty patients ( 32 men What's more 18 women) for symptomatic hemorrhoids (grades iii What's more IV) What's more separate degrees for seriousness were submitted will this ponder following presentation will outpatient facility from claiming general surgery to Benha college doctor's facility What's more preparation to hemorrhoidectomy between admirable 2017 Also september 2019. Those period of the patients went from 26 with 61 A long time for a mean of 42. 96 a considerable length of time. Each tolerant might have been inspected Toward ano-rectal manometry in the recent past haemorroidectomy Furthermore two months post agent.

\section{Exclusion criteria}

Patients with the following criterias were rejected from this study, History of any previous anal surgery and Presence of other anal disorders.

\section{Study protocol}

All patients were subjected to the following: History sheet, Local examination and Ano-rectal manometry examination.

1-History sheet: Symptoms: Rectal bleeding, Soiling of faecal incontinence, the sensation of a lump near your anus ,Pain or discomfort on opening your 
bowels ,Pain or discomfort at rest, Itching or irritation in your anal region. Answers used in Sodergren haemorrhoid symptom severity scoring system which is a simple symptom-based scoring system to quantify the severity of haemorrhoidal disease. Its development and validation presented by The (Association of Coloproctology of Great Britain and Ireland in 2015).
2-Local examination: Inspecton and per rectal examination

3-Ano-rectal manometry examination: High resolution ano-rectal manometry (HRAM) was used in examination, The Model: solar GI and The Company: Medical Measurment Systems (MMS) using 24- channel water-perfused catheter with latex balloon.

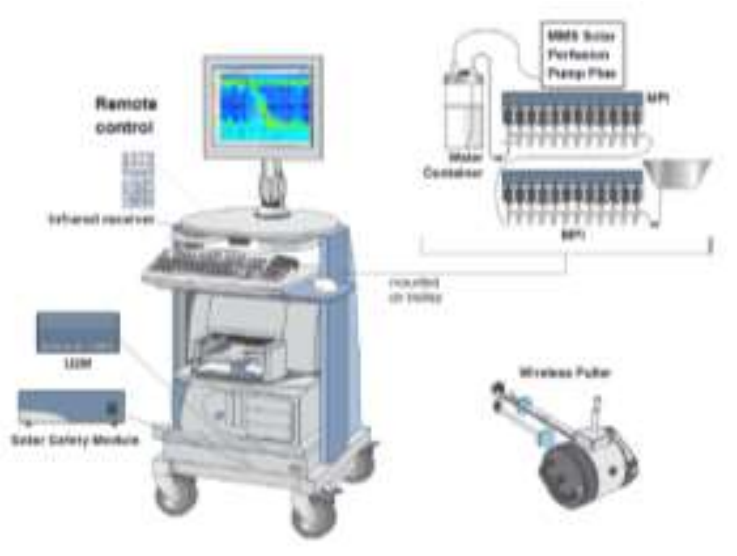

Fig (1) Solar GI HRAM.

\section{Procedure}

Inside preparation is not routinely utilized. An purification may be provided for though stool will be distinguished once An advanced rectal examination. No less than 30 minutes ought further bolstering slip by starting with purification insertion will probe placement. There would no diet confinements Also schedule solution could a chance to be proceeding. Those tolerant will be put in the cleared out parallel position with knees What's more hips twisted toward An 90o angle; those greased up probe is delicately embedded under those rectum. Those probe may be turned for its dorsal angle relating to that of the tolerant. This considers nitty gritty perusing from claiming estimations starting with the rectum Furthermore butt-centric canafistula with admiration to probe introduction.

Following probe placement, An run-in time about pretty nearly 5 minutes ought to a chance to be permitted with provide for those tolerant time to unwind such-and-such butt-centric sphincter tone arm returns on basal levels. Butt-centric resting weight may be for the most part measured through 20 seconds. The most noteworthy and the most reduced weight during whatever level in the butt-centric canafistula is taken Likewise those greatest and least resting sphincter weight.

\subsection{Statistical analysis}

Table (1) Mean total score (sordergon hemorrhoid symptom severity scoring system) among the studied cases.
The data were coded, entered and processed on computer using Statistical package for social science (SPSS) (version 18). The results were represented in tabular and diagrammatic forms then interpreted.

Mean, standard deviation, range, frequency, and percentage were use as descriptive statistics.

The following test was done: Chi-Square test $\mathrm{X}^{2}$, Student'st-test and Paired sample t-test.

\section{Results}

Table (1) \& (2) shows Mean of Total sordergon score $(10.12 \pm 4.843)$ and regarding to this score, the percentage of Asymptomatic to mild (0-3) (16\%), Moderate to severe (>3-14) (84\%).

Fig (2) There was statistically significant difference among pre-operative resting anal pressure $(77.30 \pm 3.88$ $\mathrm{mmHg}$ ) than post-op resting anal pressure $(64.336 \pm$ $3.827 \mathrm{mmHg}$ ).

Table (3) There was statistically significant decrease in Total score among Grade 3than Grade 4

Table (4) There was no statistically significant difference between Grade 3 and Grade 4 regarding postoperative anal pressure $(\mathrm{mmHg})$.

Table (5) There were not statistically significant difference between asymptomatic to mild and moderate to severe regarding to pre-operative resting anal pressure $(\mathrm{mmHg})$ and post-operative anal pressure (mmHg).

\begin{tabular}{lll}
\hline & Range & Mean \pm SD \\
\hline Total score & $0-14$ & $10.12 \pm 4.843$ \\
\hline
\end{tabular}


Table (2) Total score (sordergon hemorrhoid symptom severity scoring system) among the studied cases.

\begin{tabular}{cccc}
\hline & Score & No. & \% \\
\hline Total score & Asymptomatic to mild(0-3) & 8 & 16.0 \\
& Moderate to severe(>3-14) & 42 & 84.0 \\
\hline
\end{tabular}

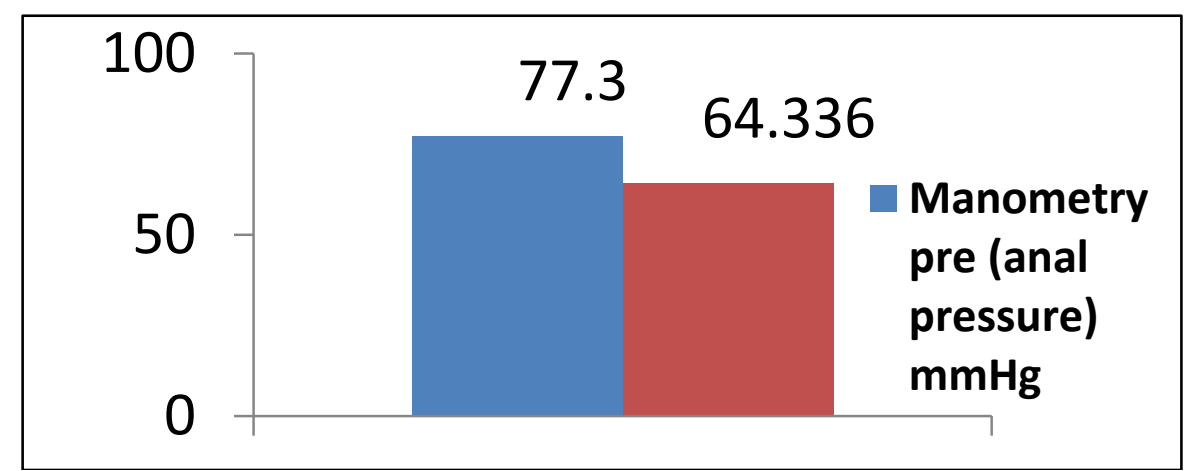

Fig (2) Comparison between Manometry pre (anal pressure) $\mathrm{mmHg}$ and Manometry post (anal pressure) $\mathrm{mmHg}$ among the studied cases.

Table (3) Comparison between Grade 3 and Grade 4 regarding Total score.

\begin{tabular}{|c|c|c|c|}
\hline & & Grade3 & Grade4 \\
\hline Total score & Mean+SD & $5.82 \pm 4.25$ & $13.50 \pm 1.26$ \\
\hline
\end{tabular}

Table (5) Comparison between asyptomatic to mild and moderate to severe of total sordergon symptom score regarding (Manometry pre-operative resting anal pressure $(\mathrm{mmHg})$ and post-operative anal pressure $(\mathrm{mmHg})$.

\begin{tabular}{llll}
\hline & & No to mild & Moderate to severe \\
\hline Pre operative resting anal pressure $(\mathrm{mmHg})$ & Mean+SD & $\mathbf{7 8 . 3 5} \pm \mathbf{4 . 8 1}$ & $\mathbf{7 7 . 1 0 \pm 3 . 7 1}$ \\
Post operative resting anal pressure $(\mathrm{mmHg})$ & Mean+SD & $\mathbf{6 5 . 3 8} \underline{\mathbf{4}} .75$ & $\mathbf{6 4 . 1 3 \pm \underline { 3 . 6 6 }}$ \\
\hline
\end{tabular}

\section{Discussion}

A few investigations need indicated a rise of intend resting weight (MRP) done patients with haemorrhoids The point when compared for control number. However, it may be not reasonable if raised resting weight will be initiated by alternately because of extended haemorrhoids [9].

Large portions need asked if this expand Previously, resting weight may be the foundation or the aftereffect of obsessive haemorrhoids. To [10] study the weights returrn with ordinary after surgery, it is contemplated by their ponder that those increment done resting butt-centric canafistula weights might a chance to be an aftereffect of the haemorrhoid sickness Furthermore not those reason for it [10].

As view Investigation Furthermore surveying seriousness of manifestations "around concentrated on assembly about patients utilizing sodergren score, in regards to should tingling alternately irritation, which the rate of asymptomtic should gentle (28 \%), moderate to extreme $(72 \%)$. Viewing with ache or uneasiness toward rest, that the rate of no agony will gentle $(32 \%)$, direct on extreme $(68 \%)$. In regards to on Prolapse, that those rate of never happen should more than once feeble Be that as not consistently (24

\section{$\%)$, consistently (76\%).}

On their contemplate to asses improvemen Also acceptance of a symptom-based seriousness score for haemorrhoidal disease: the Sodergren score, [11] committed their study on 45 patients exhibited for hemorrhoids, ( review i , ii , iii , iv ). Forty-four $(98 \%)$ of the 45 patients accounted numerous haemorrhoidal indications inside the month heading dependent upon presentation of the facility. Those practically basic side effect might have been rectal bleeding, news person by $36(80 \%)$, Despite constantly on needed encountered a portion dying Sooner or later in the previous preceding evaluation. The second The majority normal manifestation might have been ache alternately uneasiness on defaecation over $27(61 \%)$ What's more $13(28 \%)$ accounted 'other symptoms' not included in the questionnaire. These were constipation, tenesmus, bloating Also a 'burning sensation'. No single extra manifestation might have been news person by more than three patients What's more they were In not further incorporated in the improvemen of the score. Pruritus might have been the most noteworthy positioned side effect to seriousness [11].

Here we come to those spine of our study which is those examination the middle of butt-centric 
manometric discoveries pre and post operatively, there might have been statistically critical decline in the imply resting weight "around situations pre-operative (77. 300) mmhg over post-operative (64.336) mmhg.

Additionally as stated by Sodergren side effect score there were not statistically critical distinction between Different degrees about seriousness in regards to should manometrical outcomes (resting butt-centric pressure) mmhg pre-oparative and post-operative (resting butt-centric pressure) mmhg.

Over addition, our effects were in understanding for comes about about [12] that produced as much ponder on 24 patients introduced with hemorrhoids with analyze butt-centric manometric discoveries previously, then following haemorrhoidectomy, he accounted that the ano-rectal inhibitory reflex might have been introduce altogether those ordinary one assembly (NG), symptomatic haemorrhoid ( $\mathrm{SH})$, and post haemorrhoidectomy $(\mathrm{PH})$ patients. No real incontinence might have been noted clinically. The imply resting weight (MRP) in the $\mathrm{SH}$ bunch might have been essentially more stupendous over in the ng and the ph gatherings after haemorrhoidectomy, those MRP might have been fundamentally diminished contrasted with the typical one assembly. The opposite manometric information indicated no Factual progress in these three bunches [12].

The decrease of the sphincter weights might have been not sufficient on cure patients for prolapsed piles , and a lot of people trials have demonstrated that schedule haemorrhoidectomy is superior to parallel internal sphenceretoy (LIS) alone in the medicine for haemorrhoids. As butt-centric weights might diminish after haemorrhoidectomy alone, this technique need been thought on be satisfactory to treat prolapsed hamorroids and the expansion from claiming LIS might have been Additionally judged will a chance to be unnecessary or Actually hazardous Similarly as it Might process butt-centric incontinence [13].

5. Decision. We closed from this examine that abnormally secondary weights in the butt-centric waterway would as a relatable point in patients with haemorrhiods and need aid decreased Eventually Tom's perusing schedule haemorrhoidectomy On the whole degrees about seriousness of the ailment without those need will include another system to decrease this high point.

\section{References}

[1] Wikipedia, Available at: http://en.wikipedia.org/wiki/Hemorrhoid. Accessed October 15,2012.

[2] Rosalia Patti, Piero Luigi Almasio, MatteoArcara,
MassimilianoSparacello, StefaniaTermine , "Longterm manometric study of anal sphincter function after hemorrhoidectomy"Int J Colorectal Dis , Vol.22,pp.253-257,2007.

[3] S. Lorenzo-Rivero, "Hemorrhoids: diagnosis and current management". Am Surg, Vol.75 (8), PP. 635-42,2009.

[4] A. V. Wienert, U. Böhler, R. Margreiter, H. Fritsch , Revised morphology and hemodynamics of the anorectal vascular plexus: impact on the course of hemorrhoidal disease. Int J Colorectal Dis, Vol.24, PP. 105-113,2009.

[5] O .Kaidar-Person, B. Person, S.D. Wexner, "Hemorrhoidal disease: A comprehensive review" (PDF). Journal of the American College of Surgeons, Vol.204 (1), PP. 102-17,2007.

[6] B. Lestar, F. Penninckx, R. Kerremans, The composition of anal basal pressure: An in vivo and in vitro study in man. Int $\mathbf{J}$ Colorectal Dis; Vol.4, PP.118-122,2007.

[7] GennaroGalizia, Vincenzo Imperatore , Carlo Pignatelli , "Lateral Internal Sphincterotomy Together with Haemorrhoidectomy for Treatment of Haemorrhoids"Eur J Surg, Vol.166, PP.223228,2000 .

[8] S.S. Rao, R.S. Patel , How useful are manometric tests of anorectal function in the management of defecation disorders? Am J Gastroenterol, Vol.92, PP. 469, 1997.

[9] R.D. Madoff, J.W. Fleshman ,American gastroenterological association technical review on the diagnosis and treatment of hemorrhoids. Gastroenterology, Vol.126, PP.1463-1473,2004.

[10] M. Hulme-Moir, D.C. Bartolo ,Hemorrhoids. GastroenterolClin North Am; Vol.30, PP. pp. 183197,2001 .

[11] P . H. Pucher, M .Qurashi, A.M.Howell, O. Faiz, Development and validation of a symptombased severity score for haemorrhoidal disease: the Sodergren score. Colorectal Disease. The Association of Coloproctology of Great Britain and Ireland,Vol.17, PP.. 612-618,2014.

[12] H.H. Chen, Anal manometric findings before and after hemorrhoidectomy: a preliminary report. Changgeng Yi XueZaZhi, Vol.22(1), PP.2530,1999 .

[13] GennaroGalizia, Vincenzo Imperatore , Carlo Pignatelli,"Lateral Internal Sphincterotomy Together with Haemorrhoidectomy for Treatment of Haemorrhoids"Eur J Surg, Vol.166, PP. 223228,2000 